\title{
Erratum: Exact renormalization group and Sine Gordon theory
}

\author{
Prafulla Oak and B. Sathiapalan \\ Institute of Mathematical Sciences, \\ CIT Campus, Taramani, Chennai-600113, India \\ E-mail: prafullao@imsc.res.in, bala@imsc.res.in
}

ERRATUM TO: JHEP07(2017)103

ARXiv ePrint: 1703.01591

The affiliation in our paper for both authors is

- Institute of Mathematical Sciences,

CIT Campus, Taramani, Chennai-600113, India

There is another institute we are affiliated to.

Our full affiliation is

- Institute of Mathematical Sciences,

CIT Campus, Taramani, Chennai-600113, India

- Homi Bhabha National Institute,

Training School Complex, Anushaktinagar, Mumbai-400094, India

Open Access. This article is distributed under the terms of the Creative Commons Attribution License (CC-BY 4.0), which permits any use, distribution and reproduction in any medium, provided the original author(s) and source are credited. 